\title{
Genome Sequence Resource of Magnaporthe oryzae Laboratory Strain 2539
}

\author{
Meilian Chen, ${ }^{1,2}$ Baohua Wang, ${ }^{2}$ Guodong Lu, ${ }^{2}$ Zhenhui Zhong, ${ }^{2, \dagger}$ and Zonghua Wang ${ }^{1,2, \dagger}$ \\ ${ }^{1}$ Institute of Oceanography, Minjiang University, Fuzhou 350108, China \\ ${ }^{2}$ State Key Laboratory of Ecological Pest Control for Fujian and Taiwan Crops, College of Plant \\ Protection, Fujian Agriculture and Forestry University, Fuzhou 350002, China
}

\begin{abstract}
Magnaporthe oryzae causes blast disease on more than 50 species of monocot plants, including important crops such as rice, millet, and most recently wheat. Additionally, it is an important model system for studying host-pathogen interaction. Here, we report a high-quality genome assembly and annotation of a laboratory strain 2539 of $M$. oryzae, which is a widely used progeny of a rice-infecting isolate and a grass-infecting isolate. The genome sequence of strain 2539 will be useful for studying the evolution, host adaption, and pathogenicity of $M$. oryzae, which will be beneficial for a better understanding of the mechanisms of hostpathogen interaction.
\end{abstract}

\section{Genome Announcement}

Rice is one of the most important staple foods in the world. However, rice blast disease caused by Magnaporthe oryzae results in substantial economic loss to rice producers (Dean et al. 2012). Because of its high genome plasticity and host adaption, M. oryzae can infect more than 50 species of plants, such as wheat, oat, finger millet, and some graminaceous weeds that grow near the cultivated crops (Ou 1985). In addition, M. oryzae is an important model system for studying host-pathogen interaction (Ebbole 2007). Presently, genome sequence data for numerous $M$. oryzae strains are available that greatly enhanced our understanding of essential traits, including host specificity, host adaption, and global epidemiology.

M. oryzae 2539 is a laboratory strain widely used for telomere analysis, avirulence (AVR) gene cloning, identification of transposon elements, and plant immunity micro-RNA research (Kachroo et al. 1995; Starnes et al. 2012; Wang et al. 2018; Zhang et al. 2018; Zheng et al. 2011). Strain 2539 was a single spore isolated from a single lesion on rice inoculated with an ascus culture from cross $\mathrm{CH} 40-1 \times 4162$ (Leung et al. 1988). CH40-1 is a M. grisea strain that is highly virulent on nine rice cultivars (Kolmer and Ellingboe 1988). Previous studies found that 736 of 765 tested rice lines could not be infected by strain 2539, suggesting strain 2539 likely carries some predominant AVR genes (Smith and Leong 1994). Additional studies also showed that AVR1-CO39 was one of the predominant AVR genes controlling the broad avirulence of strain 2539 against rice (Zheng et al. 2011). However, the genome sequence of this strain is still not available.

In this study, the genomic DNA of strain 2539 was extracted and purified from mycelium and was sequenced with the paired-end Illumina HiSeq2500 platform. We obtained 6.796

†Corresponding authors: Z. Wang; wangzh@fafu.edu.cn and Z. Zhong; zhenhuizhong@gmail.com

The author(s) declare no conflict of interest.

Accepted for publication 27 April 2020.

(C) 2020 The American Phytopathological Society

\section{Funding}

This work was supported by grants from the National Natural Science Foundation of China (U1805232, $31770156)$ and the National Key R\&D Program of China (2016YFD0300700) to Z. Wang.

\section{Keywords}

genome plasticity, host adaption, host-pathogen interaction,

Magnaporthe oryzae laboratory strain 2539, monocots 
Table 1. Genome features of Magnaporthe oryzae 2539

\begin{tabular}{ll} 
Variables & \multicolumn{1}{c}{ Statistics } \\
Mating type & Mat1-1 \\
Sequence coverage & $34 \times$ \\
Genome size (Mb) & 38.08 \\
Contigs (count) & 1,391 \\
Contig N $N_{50}$ size (bp) & 107,099 \\
Max contig size (bp) & 503,659 \\
Average contig size (bp) & 27,378 \\
GC content (\%) & 51.1 \\
Number of predicted genes & 12,116 \\
Secreted proteins & 1,051 \\
BUSCO completeness (\%) & 98.5 \\
Genome accession & VCMT00000000 \\
\hline
\end{tabular}

million 100-bp reads with 500-bp insert length, which represents 34-fold genome coverage. A 38.08 Mb genome assembly was obtained with CLC genomic workbench (v12.0.1; Qiagen). This is closer to the genome size predicted with Kmergenie (38.13 $\mathrm{Mb}$ ) and similar to the published genome sequences of $M$. oryzae strains sequenced with short-read sequencing while smaller than strains sequenced with long-read sequencing (Bao et al. 2017; Chikhi and Medvedev 2014; Gómez Luciano et al. 2019; Zhong et al. 2016, 2018). GC content of strain 2539 is $51.1 \%$, which is also close to isolates of the $M$. oryzae species (Zhong et al. 2016). The draft genome sequence of strain 2539 includes 1,391 contigs $\left(\mathrm{N}_{50} 107 \mathrm{~Kb}\right)$ and an average contig size of $27 \mathrm{~Kb}$ (Table 1). The completeness of strain 2539 assembly was evaluated with BUSCO (v3.0.2) with 'Ascomycota odb9' as a reference, based on a dataset of near-universal single-copy orthologous genes of 1,315 common Ascomycota genes (Waterhouse et al. 2017). This analysis indicates strain 2539 is a genome with high completeness ( $98.5 \%$ complete).

Protein-coding genes were predicted by conducting through a combination of evidencebased prediction by Exonerate (v2.2.0) with M. oryzae 70-15 genes as reference and ab initio prediction with Fgenesh with Magnaporthe as training organism (Dean et al. 2005). Our prediction identified a total of 12,116 protein-coding genes. Secreted proteins, as used in this study, refer to proteins containing a N-terminal signal peptide cleavage site (predicted with SignalP 4.0) and with no transmembrane domain after the region signal peptide cleavage site (predicted with TMHMM 2.0) (Krogh et al. 2001; Petersen et al. 2011). With this method, we identified 1,051 secreted proteins in strain 2539. Gene functions were annotated with HMMER (v 3.1) (Finn et al. 2011). Genome-wide gene comparison between strain 2539 and strain 70-15 revealed a total of 748 genes are present in the 2539 genome but may be absent in the 70-15 genome. Putative carbohydrate-active enzymes (CAZymes) were predicted using dbCAN (Yin et al. 2012). We identified a total of 738 CAZymes, including 105 auxiliary activity family proteins, 274 glycoside hydrolases, 103 glycosyltransferases, five polysaccharide lyases, 129 carbohydrate esterases, and 122 noncatalytic carbohydrate-binding modules. With GPCRHMM, we also recorded $51 \mathrm{G}$ protein-coupled receptors (GPCRs) in the genome of strain 2539 compared with 56 recorded for the 70-15 genome (Wistrand et al. 2006). Additionally, we annotated AVR genes in the strain 2539 genome and found that the 2539 genome contains Avr-Pi54, Avr1-CO39, AvrPi9, AvrPib, AvrPiz-t, and ACE1, while AvrPik, AvrPia, AvrPii, AvrPita, PWL1, and PWL2 are absent in the genome.

Assembled contigs are available at the National Center for Biotechnology Information under BioProject ID PRJNA544882, BioSample ID SAMN11866126. The genome version described in this paper is VCMT00000000. The genome annotation file and lists of secreted proteins, unique genes, CAZymes, GPCRs, and AVRs are available in Figshare (Chen et al. 2020).

\section{Literature Cited}

Bao, J., Chen, M., Zhong, Z., Tang, W., Lin, L., Zhang, X., Jiang, H., Zhang, D., Miao, C., Tang, H., Zhang, J., Lu, G., Ming, R., Norvienyeku, J., Wang, B., and Wang, Z. 2017. PacBio sequencing reveals transposable elements as a key contributor to genomic plasticity and virulence variation in Magnaporthe oryzae. Mol. Plant 10:1465-1468.
Chen, M., Wang, B., Lu, G., Zhong, Z., and Wang, Z. 2020. Genome sequence resource of Magnaporthe oryzae laboratory strain 2539. figshare https://doi.org/10.6084/ m9.figshare.11849121.v1. Published online.

Chikhi, R., and Medvedev, P. 2014. Informed and automated k-mer size selection for genome assembly. Bioinformatics 30:31-37.

Dean, R., Van Kan, J. A., Pretorius, Z. A., Hammond-Kosack, K. E., Di Pietro, A., Spanu, P. D., Rudd, J. J., Dickman, M., Kahmann, R., Ellis, J., and Foster, G. D. 
2012. The top 10 fungal pathogens in molecular plant pathology. Mol. Plant Pathol. 13:414-430.

Dean, R. A., Talbot, N. J., Ebbole, D. J., Farman, M. L., Mitchell, T. K., Orbach, M. J., Thon, M., Kulkarni, R., Xu, J. R., Pan, H., Read, N. D., Lee, Y. H., Carbone, I., Brown, D., Oh, Y. Y., Donofrio, N., Jeong, J. S., Soanes, D. M., Djonovic, S., Kolomiets, E., Rehmeyer, C., Li, W., Harding, M., Kim, S., Lebrun, M. H., Bohnert, H., Coughlan, S., Butler, J., Calvo, S., Ma, L. J., Nicol, R., Purcell, S., Nusbaum, C., Galagan, J. E., and Birren, B. W. 2005. The genome sequence of the rice blast fungus Magnaporthe grisea. Nature 434:980-986.

Ebbole, D. J. 2007. Magnaporthe as a model for understanding host-pathogen interactions. Annu. Rev. Phytopathol. 45:437-456.

Finn, R. D., Clements, J., and Eddy, S. R. 2011. HMMER web server: Interactive sequence similarity searching. Nucleic Acids Res. 39:W29-W37.

Gómez Luciano, L. B., Tsai, I. J., Chuma, I., Tosa, Y., Chen, Y.-H., Li, J.-Y., Li, M.-Y., Lu, M. J., Nakayashiki, H., and Li, W.-H. 2019. Blast fungal genomes show frequent chromosomal changes, gene gains and losses, and effector gene turnover. Mol. Biol. Evol. 36:1148-1161.

Kachroo, P., Leong, S. A., and Chattoo, B. B. 1995. Mg-SINE: A short interspersed nuclear element from the rice blast fungus, Magnaporthe grisea. Proc. Natl. Acad. Sci. U.S.A. 92:11125-11129.

Kolmer, J. A., and Ellingboe, A. H. 1988. Genetic relationships between fertility and pathogenicity and virulence to rice in Magnaporthe grisea. Can. J. Bot. 66: 891-897.

Krogh, A., Larsson, B., von Heijne, G., and Sonnhammer, E. L. 2001. Predicting transmembrane protein topology with a hidden Markov model: Application to complete genomes. J. Mol. Biol. 305:567-580.

Leung, H., Borromeo, E. S., Bernardo, M. A., and Notteghem, J. L. 1988. Genetic analysis of virulence in the rice blast fungus Magnaporthe grisea. Phytopathology 78:1227-1233.

Ou, S. H. 1985. Rice Diseases. 2nd ed. Commonwealth Mycological Institute, Kew, U.K.

Petersen, T. N., Brunak, S., von Heijne, G., and Nielsen, H. 2011. SignalP 4.0: Discriminating signal peptides from transmembrane regions. Nat. Methods 8:785-786.

Smith, J. R., and Leong, S. A. 1994. Mapping of a Magnaporthe grisea locus affecting rice (Oryza sativa) cultivar specificity. Theor. Appl. Genetics 88:901-908.
Starnes, J. H., Thornbury, D. W., Novikova, O. S., Rehmeyer, C. J., and Farman, M. L. 2012. Telomere-targeted retrotransposons in the rice blast fungus Magnaporthe oryzae: Agents of telomere instability. Genetics 191:389-406.

Wang, J., Guo, X., Li, L., Qiu, H., Zhang, Z., Wang, Y., and Sun, G. 2018. Application of the fluorescent dye BODIPY in the study of lipid dynamics of the rice blast fungus Magnaporthe oryzae. Molecules 23:1594.

Waterhouse, R. M., Seppey, M., Simao, F. A., Manni, M., loannidis, P., Klioutchnikov, G., Kriventseva, E. V., and Zdobnov, E. M. 2017. BUSCO applications from quality assessments to gene prediction and phylogenomics. Mol. Biol. Evol 35: 543-548..

Wistrand, M., Käll, L., and Sonnhammer, E. L. 2006. A general model of G proteincoupled receptor sequences and its application to detect remote homologs. Protein Sci. 15:509-521.

Yin, Y., Mao, X., Yang, J., Chen, X., Mao, F., and Xu, Y. 2012. dbCAN: A web resource for automated carbohydrate-active enzyme annotation. Nucleic Acids Res. 40:W445-W451.

Zhang, X., Bao, Y., Shan, D., Wang, Z., Song, X., Wang, Z., Wang, J., He, L., Wu, L., Zhang, Z., Niu, D., Jin, H., and Zhao, H. 2018. Magnaporthe oryzae induces the expression of a microRNA to suppress the immune response in rice. Plant Physiol. 177:352-368.

Zheng, Y., Zheng, W., Lin, F., Zhang, Y., Yi, Y., Wang, B., Lu, G., Wang, Z., and Wu, W. 2011. AVR1-CO39 is a predominant locus governing the broad avirulence of Magnaporthe oryzae 2539 on cultivated rice (Oryza sativa L.). Mol. PlantMicrobe Interact. 24:13-17.

Zhong, Z., Chen, M., Lin, L., Han, Y., Bao, J., Tang, W., Lin, L., Lin, Y., Somai, R., Lu, L., Zhang, W., Chen, J., Hong, Y., Chen, X., Wang, B., Shen, W. C., Lu, G., Norvienyeku, J., Ebbole, D. J., and Wang, Z. 2018. Population genomic analysis of the rice blast fungus reveals specific events associated with expansion of three main clades. ISME J. 12:1867-1878.

Zhong, Z., Norvienyeku, J., Chen, M., Bao, J., Lin, L., Chen, L., Lin, Y., Wu, X., Cai, Z., Zhang, Q., Lin, X., Hong, Y., Huang, J., Xu, L., Zhang, H., Chen, L., Tang, W., Zheng, H., Chen, X., Wang, Y., Lian, B., Zhang, L., Tang, H., Lu, G., Ebbole, D. J., Wang, B., and Wang, Z. 2016. Directional selection from host plants is a major force driving host specificity in Magnaporthe species. Sci. Rep. 6:25591. 